\title{
HARMONIC SPHERES IN THE HILBERT-SCHMIDT GRASSMANNIAN
}

\author{
IULIYA BELOSHAPKA, ARMEN SERGEEV \\ To Sergei Petrovich Novikov on the occasion of his 75th birthdate
}

\begin{abstract}
We give the twistor description of harmonic maps of the Riemann sphere into the Hilbert-Schmidt Grassmannian. The study of such maps is motivated by the harmonic spheres conjecture formulated in the beginning of this paper.
\end{abstract}

\section{INTRODUCTION}

A well known theorem of Atiyah [2] establishes a 1-1 correspondence between the moduli space of $G$-instantons on 4-dimensional Euclidean space $\mathbb{R}^{4}$ and the space of based holomorphic maps of the Riemann sphere $\mathbb{P}^{1}$ into the loop space $\Omega G$ of a compact Lie group $G$. The harmonic spheres conjecture, obtained from this formulation by "realification", asserts that it should exist a natural 1-1 correspondence between the moduli space of Yang-Mills $G$-fields on $\mathbb{R}^{4}$ and the space of based harmonic maps of the Riemann sphere $\mathbb{P}^{1}$ into the loop space $\Omega G$.

The proof of the Atiyah theorem depends on the Donaldson theorem [7], establishing a 1-1 correspondence between the moduli space of $G$-instantons on $\mathbb{R}^{4}$ and the set of equivalence classes of holomorphic $G^{\mathbb{C}}$-bundles over $\mathbb{P}^{1} \times \mathbb{P}^{1}$ which are trivial on the union $\mathbb{P}_{\infty}^{1} \cup \mathbb{P}_{\infty}^{1}$ of projective lines at "infinity". The Donaldson theorem may be considered as a 2-dimensional reduction of the well known Atiyah-Ward theorem, relating instantons on $\mathbb{R}^{4}$ with holomorphic bundles on the 3 -dimensional projective space $\mathbb{P}^{3}$ which are trivial on the fibres of the twistor bundle $\mathbb{P}^{3} \backslash \mathbb{P}_{\infty}^{1} \rightarrow \mathbb{R}^{4}$. The proof of Donaldson theorem is based on the monad method, used for the construction of holomorphic vector bundles on projective spaces, and is "purely complex". So to prove the harmonic spheres conjecture one should look for a "real" analogue of Donaldson theorem for harmonic bundles.

1991 Mathematics Subject Classification. Primary 58E20, 53C28,32L25.

While preparing this paper the second author was partly supported by the RFBR grant 13-01-00622, the Leading Scientific Schools program (grant NSh-2928.2012.1), and Scientific Program of Presidium of RAS "Nonlinear dynamics". 
Such an analogue was proposed in [15] where it was also outlined an idea of the proof of the harmonic spheres conjecture. One of its essential ingredients is the twistor description of harmonic spheres in the Hilbert-Schmidt Grassmannian which is the main subject of this paper.

\section{Motivation: HARMOniC SPHERES CONJECTURE}

The motivation to study harmonic maps of the Riemann sphere into the Hilbert-Schmidt Grassmannian comes from the harmonic spheres conjecture which is formulated below.

We start by recalling some necessary facts about instantons and Yang-Mills fields on the one hand, and harmonic maps on the other hand.

1.1. Instantons and Yang-Mills fields. Let $G$ be a compact Lie group and $A$ is a $G$-connection on $\mathbb{R}^{4}$ given by the 1 -form

$$
A=\sum_{\mu=1}^{4} A_{\mu}(x) d x_{\mu}
$$

with smooth coefficients $A_{\mu}(x)$, taking values in the Lie algebra $\mathfrak{g}$ of $G$. Denote by $F_{A}$ the curvature of $A$ given by the 2 -form

$$
F_{A}=\sum_{\mu, \nu=1}^{4} F_{\mu \nu}(x) d x_{\mu} \wedge d x_{\nu}
$$

with coefficients

$$
F_{\mu \nu}=\partial_{\mu} A_{\nu}-\partial_{\nu} A_{\mu}+\left[A_{\mu}, A_{\nu}\right]
$$

where $\partial_{\mu}:=\partial / \partial x_{\mu}, \mu=1,2,3,4$, and $[\cdot, \cdot]$ denotes the commutator in Lie algebra $\mathfrak{g}$.

Define the Yang-Mills action functional by the formula

$$
S(A)=\frac{1}{2} \int_{\mathbb{R}^{4}} \operatorname{tr}\left(F_{A} \wedge * F_{A}\right)
$$

where $*$ is the Hodge operator on $\mathbb{R}^{4}$, and the trace tr is computed with the help of a fixed invariant inner product on the Lie algebra $\mathfrak{g}$.

The functional $S(A)$ is invariant under the gauge transformations given by

$$
A \longmapsto A_{g}:=g^{-1} d g+g^{-1} A g
$$

where $g: \mathbb{R}^{4} \rightarrow G$ is a smooth map, and $G$ acts on its Lie algebra $\mathfrak{g}$ by the adjoint representation.

The extremals of the functional $S(A)$ with finite action $S(A)<\infty$ are called the Yang-Mills fields. 
Yang-Mills fields have an integer-valued topological invariant, called the topological charge, computed by the formula

$$
k(A)=\frac{1}{8 \pi^{2}} \int_{\mathbb{R}^{4}} \operatorname{tr}\left(F_{A} \wedge F_{A}\right) .
$$

If we write $F_{A}$ in the form

$$
F_{A}=F_{+}+F_{-}
$$

where $F_{ \pm}=\frac{1}{2}\left(* F_{A} \pm F_{A}\right)$ then the formulae for the action and charge may be rewritten as

$$
\begin{aligned}
& S(A)=\frac{1}{2} \int_{\mathbb{R}^{4}}\left(\left\|F_{+}\right\|^{2}+\left\|F_{-}\right\|^{2}\right) d^{4} x, \\
& k(A)=\frac{1}{8 \pi^{2}} \int_{\mathbb{R}^{4}}\left(-\left\|F_{+}\right\|^{2}+\left\|F_{-}\right\|^{2}\right) d^{4} x
\end{aligned}
$$

where the norm $\|\cdot\|^{2}$ is computed with the help of invariant inner product on the Lie algebra $\mathfrak{g}$.

By comparing the last two formulae we arrive at the inequality

$$
S(A) \geq 4 \pi^{2}|k(A)|
$$

where the equality is attained for $k>0$ on solutions of the equation

$$
* F_{A}=-F_{A}
$$

and for $k<0$ on solutions of the equation

$$
* F_{A}=F_{A} \text {. }
$$

Definition 1. Solutions of equation (11) with $S(A)<\infty$ are called instantons, and solutions of equation (2) with $S(A)<\infty$ are called anti-instantons.

Instantons and anti-instantons realize local minima of the action $S(A)$, however, there exist also non-minimal critical points of this functional.

1.2. Harmonic spheres. Let $\varphi: \mathbb{P}^{1} \rightarrow N$ be a smooth map from the Riemann sphere $\mathbb{P}^{1}$ into an oriented Riemannian manifold $N$. We call such a map harmonic if it is extremal with respect to the energy functional given by the following Dirichlet integral

$$
E(\varphi)=\frac{1}{2} \int_{\mathbb{C}}|d \varphi|_{N}^{2} \frac{|d z \wedge d \bar{z}|}{\left(1+|z|^{2}\right)^{2}}
$$

where the modulus of differential $d \varphi$ is computed with respect to the metric $|\cdot|_{N}$ of manifold $N$.

If the manifold $N$ is Kähler, i.e. $N$ has a complex structure, compatible with the Riemannian metric, then holomorphic and anti-holomorphic maps 
$\varphi: \mathbb{P}^{1} \rightarrow N$ realize local minima of the energy functional $E(\varphi)$. However, for $\operatorname{dim}_{\mathbb{C}} N>1$ there exist usually also non-minimal harmonic maps.

Comparing harmonic maps with Yang-Mills fields, we notice an evident analogy between:

$$
\{(\text { anti)holomorphic maps }\} \longleftrightarrow\{(\text { anti)instantons }\}
$$

and

$$
\{\text { harmonic maps }\} \longleftrightarrow\{\text { Yang-Mills fields }\} .
$$

We are going to give a mathematical justification of this observation.

1.3. Twistor interpretation of instantons. The twistor interpretation of instantons is based on the following twistor bundle

$$
\pi: \mathbb{P}^{3} \backslash \mathbb{P}_{\infty}^{1} \longrightarrow \mathbb{R}^{4}
$$

over the Euclidean space $\mathbb{R}^{4}$ where $\mathbb{P}^{3}$ is the 3 -dimensional complex projective space (cf. [1]). The fibre of this bundle at $x \in \mathbb{R}^{4}$ can be identified with the space of complex structures on the tangent space $T_{x} \mathbb{R}^{4} \cong \mathbb{R}^{4}$, compatible with metric and orientation.

In terms of the twistor bundle $\pi: \mathbb{P}^{3} \backslash \mathbb{P}^{1} \rightarrow \mathbb{R}^{4}$ the moduli space of $G$ instantons, i.e. the quotient of the space of all $G$-instantons on $\mathbb{R}^{4}$ modulo gauge transformations, admits the following interpretation given by the Atiyah-Ward theorem:

$\left\{\begin{array}{l}\text { moduli space of } G \text { - } \\ \text { instatons on } \mathbb{R}^{4}\end{array}\right\} \longleftrightarrow\left\{\begin{array}{l}(\text { based) equivalence classes of holomorphic } \\ G^{\mathbb{C}} \text {-bundles over } \mathbb{P}^{3} \text {, holomorphically trivial } \\ \text { on } \pi \text {-fibers }\end{array}\right\}$.

This result has the following 2 -dimensional reduction to the space $\mathbb{P}^{1} \times \mathbb{P}^{1}$ given by the Donaldson theorem:

$\left\{\begin{array}{l}\text { moduli space of } G- \\ \text { instantons on } \mathbb{R}^{4}\end{array}\right\} \longleftrightarrow\left\{\begin{array}{l}\left(\text { based) equivalence classes of holomorphic } G^{\mathbb{C}}-\right. \\ \text { bundles over } \mathbb{P}^{1} \times \mathbb{P}^{1}, \text { holomorphically trivial on } \\ \text { the union } \mathbb{P}_{\infty}^{1} \cup \mathbb{P}_{\infty}^{1}\end{array}\right\}$.

1.4. Twistor interpretation of harmonic spheres. Using the interpretation of the twistor bundle over $\mathbb{R}^{4}$ as the bundle of compatible complex structure, one can extend its definition to any even-dimensional oriented Riemannian manifold $N$. Namely, the twistor bundle $\pi: Z \rightarrow N$ over $N$ is defined as the bundle of complex structures on $N$, compatible with metric and orientation. The fibre of this bundle can be identified with the homogeneous space $\mathrm{SO}(2 n) / \mathrm{U}(n)$ where $2 n$ is the dimension of $N$. The twistor space $Z$ can be provided, as it was shown in [3], with a natural almost complex structure, denoted by $\mathcal{J}^{1}$. 
However, for the description of harmonic spheres in $N$ we have to employ another almost complex structure which is defined in the following way. The Levi-Civita connection on $N$ generates a connection on the twistor bundle $\pi: Z \rightarrow N$. The new almost complex structure on $Z$, denoted by $\mathcal{J}^{2}$, is equal to $\mathcal{J}^{1}$ in the directions, horizontal with respect to the introduced connection, and to $-\mathcal{J}^{1}$ in vertical directions. This structure was introduced in [8] and is always non integrable. Harmonic spheres in $N$ admit the following description in terms of this almost complex structure.

Theorem 1 (Eells-Salamon [8]). Projections $\varphi=\pi \circ \psi$ of the maps $\psi: \mathbb{P}^{1} \rightarrow$ $Z$, holomorphic with respect to the almost complex structure $\mathcal{J}^{2}$, are harmonic.

This theorem allows to construct harmonic spheres in the manifold $N$ from almost holomorphic spheres in its twistor space $Z$. And this is an idea of the twistor approach to harmonic maps - to try to reduce the original "real" problem of construction of harmonic spheres in the Riemannian manifold $N$ to the "complex" problem of construction of holomorphic spheres in the almost complex manifold $Z$.

We show in Section 2.2 how this idea can be applied to the description of harmonic spheres in the complex Grassmann manifold $G_{r}\left(\mathbb{C}^{d}\right)$.

1.5. Atiyah theorem. We switch now to the case when the target Riemannian manifold $N$ is infinite-dimensional, more precisely, the loop space of a compact Lie group.

Recall first the definition of this space. Let $G$ be a compact Lie group. Consider the quotient

$$
\Omega G:=L G / G
$$

of the loop group $L G=C^{\infty}\left(S^{1}, G\right)$ modulo the subgroup of constant maps $S^{1} \rightarrow g_{0} \in G$ identified with the group $G$. The space $\Omega G$ is a Frechet manifold which has an $L G$-invariant complex structure (cf. [12]). This structure is induced from the representation of $\Omega G$ as the quotient of the complex loop group $L G^{\mathbb{C}}$ :

$$
\Omega G=L G^{\mathbb{C}} / L_{+} G^{\mathbb{C}}
$$

where $G^{\mathbb{C}}$ is the complexification of the group $G$ and the subgroup $L_{+} G^{\mathbb{C}}$ consists of the loops $\gamma \in L G^{\mathbb{C}}$ which can be smoothly extended to holomorphic maps of the unit disc $\Delta$ into $G^{\mathbb{C}}$. Apart from the complex structure $\Omega G$ has also an $L G$-invariant symplectic structure (cf. [12]) which is compatible with the introduced complex structure in the sense that they generate together a natural Riemannian metric on $\Omega G$. 
Recall that by Donalson theorem the moduli space of $G$-instantons can be identified with:

$\left\{\begin{array}{l}\text { moduli space of } G \text { - } \\ \text { instantons on } \mathbb{R}^{4}\end{array}\right\} \longleftrightarrow\left\{\begin{array}{l}\text { (based) equivalence classes of holomorphic } G^{\mathbb{C}_{-}} \\ \text {bundles over } \mathbb{P}^{1} \times \mathbb{P}^{1} \text {, holomorphically trivial on } \\ \text { the union } \mathbb{P}_{\infty}^{1} \cup \mathbb{P}_{\infty}^{1}\end{array}\right\}$

Atiyah theorem asserts that the right hand side of this correspondence may be identified with the space of holomorphic spheres in $\Omega G$. In more detail, there is a $1-1$ correspondence between:

$$
\left\{\begin{array}{l}
\text { (based) equivalence classes of } \\
\text { holomorphic } G^{\mathbb{C}} \text {-bundles over } \\
\mathbb{P}^{1} \times \mathbb{P}^{1} \text {, holomorphically trivial } \\
\text { on the union } \mathbb{P}_{\infty}^{1} \cup \mathbb{P}_{\infty}^{1}
\end{array}\right\} \longleftrightarrow\left\{\begin{array}{l}
\text { based holomorphic spheres } f: \\
\mathbb{P}^{1} \rightarrow \Omega G \text {, sending } \infty \text { into the } \\
\text { origin of } \Omega G
\end{array}\right\} .
$$

1.6. Harmonic spheres conjecture. The Donaldson and Atiyah theorems imply that there is a 1-1 correspondence between:

$$
\left\{\begin{array}{l}
\text { moduli space of } G \text { - } \\
\text { instantons on } \mathbb{R}^{4}
\end{array}\right\} \longleftrightarrow\left\{\begin{array}{l}
\text { based holomorphic spheres } \\
f: \mathbb{P}^{1} \rightarrow \Omega G
\end{array}\right\} .
$$

So we have a correspondence between local minima of two functionals, introduced before, namely the Yang-Mills action, defined on gauge $G$-fields on $\mathbb{R}^{4}$, and the energy functional, defined on smooth spheres in the loop space $\Omega G$. Recall that the local minima of Yang-Mills action are given by instantons and anti-instantons on $\mathbb{R}^{4}$ while the local minima of energy are given by holomorphic and anti-holomorphic spheres in $\Omega G$. Replacing local minima by critical points of these functionals, we arrive at the harmonic spheres conjecture asserting that it should exist a 1-1 correspondence between:

$$
\left\{\begin{array}{l}
\text { moduli space of Yang-Mills } \\
G \text {-fields on } \mathbb{R}^{4}
\end{array}\right\} \longleftrightarrow\left\{\begin{array}{l}
\text { based harmonic spheres } f: \\
\mathbb{P}^{1} \rightarrow \Omega G
\end{array}\right\} .
$$

The described transition from the local minima to critical points may be considered as a kind of the "realification" procedure. Indeed, if we replace smooth spheres in the right hand side of the diagram by smooth functions $f: \mathbb{C} \rightarrow \mathbb{C}$ then the above procedure will reduce to the replacement of holomorphic and anti-holomorphic functions by arbitrary harmonic functions (which can be represented as sums of holomorphic and anti-holomorphic functions). In the case of smooth spheres in $\Omega G$ this transition from holomorphic and anti-holomorphic spheres to harmonic ones becomes non-trivial due to the non-linear character of the Euler-Lagrange equations for the energy.

Unfortunately, a direct generalization of Atiyah-Donaldson theorem to the harmonic case is not possible since the proof of Donaldson theorem, using the monad method, is purely holomorphic and does not extend directly to the 
harmonic case. However, in [15] it was proposed another way of proving the formulated conjecture. Namely, one can try to reduce the proof of harmonic spheres conjecture to the holomorphic case by "pulling-up" the both sides of the correspondence in this conjecture to the associated twistor spaces.

One of the key points in this approach is an extension of the twistor construction of harmonic maps, presented in Sec. 1.4, to the case of infinite-dimensional target spaces. More precisely, we are interested in the infinite-dimensional generalization of the construction of harmonic maps into the complex Grassmann manifold $G_{r}\left(\mathbb{C}^{d}\right)$, presented in Section 2.2.

\section{Harmonic maps into COMPlex Grassmanninans}

In this Section we study harmonic maps from the Riemann surfaces into the complex Grassmann manifolds $G_{r}\left(\mathbb{C}^{d}\right)$.

2.1. Harmonic maps into Riemannian manifolds. Recall first some general properties of harmonic maps of Riemannian manifolds.

Let $\varphi:(M, g) \rightarrow(N, h)$ be a smooth map of a Riemannian manifold $M$ with Riemannian metric $g$ into a Riemannian manifold $N$ with Riemannian metric $h$. We define the energy of the map $\varphi$ as the Dirichlet integral

$$
E(\varphi)=\frac{1}{2} \int_{M}|d \varphi(p)|^{2} \operatorname{vol}_{g}
$$

The norm of the differential may be computed in local coordinates as follows. Denote by $\left(x^{i}\right)$ local coordinates at $p \in M$ and by $\left(u^{\alpha}\right)$ local coordinates at $q=\varphi(p) \in N$. Then

$$
|d \varphi(p)|^{2}=\sum_{i, j} \sum_{\alpha, \beta} g^{i j} \frac{\partial \varphi^{\alpha}}{\partial x^{i}} \frac{\partial \varphi^{\beta}}{\partial x^{j}} h_{\alpha \beta}
$$

where $\varphi^{\alpha}=\varphi^{\alpha}(x)$ are the components of $\varphi,\left(g_{i j}\right)$ and $\left(h_{\alpha \beta}\right)$ are metric tensors of $M$ and $N$ respectively, and $\left(g^{i j}\right)$ is the inverse matrix of $\left(g_{i j}\right), \operatorname{vol}_{g}$ is the volume element of metric $g$.

A smooth map $\varphi: M \rightarrow N$ is called harmonic if it is extremal for the functional $E(\varphi)$ with respect to all smooth variations of $\varphi$ with compact supports.

The Euler-Lagrange equation for the energy functional $E(\varphi)$, called otherwise the harmonic map equation, has the following form in local coordinates $\left(x^{i}\right)$ on $M$ and $\left(u^{\alpha}\right)$ on $N$ :

$$
\Delta_{M} \varphi^{\gamma}+\sum_{i, j} g^{i j} \sum_{\alpha, \beta}{ }^{N} \Gamma_{\alpha \beta}^{\gamma}(\varphi) \frac{\partial \varphi^{\alpha}}{\partial x_{i}} \frac{\partial \varphi^{\beta}}{\partial x_{j}}=0
$$


where $\Delta_{M}$ is the standard Laplace-Beltrami operator on $M$, given by

$$
\Delta_{M} \varphi^{\gamma}=\sum_{i, j} g^{i j}\left\{\frac{\partial^{2} \varphi^{\gamma}}{\partial x_{i} \partial x_{j}}-\sum_{k}{ }^{M} \Gamma_{i j}^{k} \frac{\partial \varphi^{\gamma}}{\partial x_{k}}\right\} .
$$

Here, ${ }^{M} \Gamma_{i j}^{k}$ denotes the Christoffel symbol of the Levi-Civita connection ${ }^{M} \nabla$ of $M$; respectively, ${ }^{N} \Gamma_{\alpha \beta}^{\gamma}$ is the Christoffel symbol of the Levi-Civita connection ${ }^{N} \nabla$ of $N$.

In particular case $N=\mathbb{R}^{n}$ the equation (5) becomes linear and reduces to the Laplace-Beltrami equation

$$
\Delta_{M} \varphi^{\gamma}=0, \quad \gamma=1, \ldots, n
$$

on the components of $\varphi$.

Suppose that the Riemannian manifold $(M, g)$ is provided with a complex (or almost complex) structure ${ }^{M} J$, compatible with Riemannian metric $g$, and, likewise, the target manifold $(N, h)$ has a complex (or almost complex) structure ${ }^{N} J$, compatible with Riemannian metric $h$.

Recall that a smooth map $\varphi: M \rightarrow N$ is called (pseudo)holomorphic if the tangent map $\varphi_{*}: T M \rightarrow T N$ commutes with (almost) complex structures on $M$ and $N$, i.e.

$$
\varphi_{*} \circ{ }^{M} J={ }^{N} J \circ \varphi_{*},
$$

and it is called anti-(pseudo)holomorphic if $\varphi_{*}$ anti-commutes with the (almost) complex structures on $M$ and $N$.

The complexified tangent bundle $T^{\mathbb{C}} M=T M \otimes \mathbb{C}$ can be decomposed into the direct sum

$$
T^{\mathbb{C}} M=T^{1,0} M \oplus T^{0,1} M
$$

of subbundles with the fibres, given by the $( \pm i)$-eigenspaces of the almost complex structure operator ${ }^{M} J$. If we extend the tangent map $\varphi_{*}$ complex linearly to the complexified tangent bundles, then we obtain a map $\varphi_{*}: T^{\mathbb{C}} M \rightarrow T^{\mathbb{C}} N$, which, in accordance with the above decomposition, splits into the sum of four operators

$$
\begin{aligned}
\partial^{\prime} \varphi: T^{1,0} M \rightarrow T^{1,0} N \quad, \quad \partial^{\prime \prime} \varphi: T^{0,1} M \rightarrow T^{1,0} N \\
\partial^{\prime} \bar{\varphi}=\overline{\partial^{\prime \prime} \varphi}: T^{1,0} M \rightarrow T^{0,1} N \quad, \quad \partial^{\prime \prime} \bar{\varphi}=\overline{\partial^{\prime} \varphi}: T^{0,1} M \rightarrow T^{0,1} N .
\end{aligned}
$$

The introduced operators may be considered as sections of the bundle $\left(T^{*} M\right)^{\mathbb{C}} \otimes$ $\varphi^{-1}\left(T^{\mathbb{C}} N\right)$. In these notations the map $\varphi$ is pseudoholomorphic (resp. antipseudoholomorphic) if and only if $\partial^{\prime \prime} \varphi=0\left(\right.$ resp. $\left.\partial^{\prime} \varphi=0\right)$.

It may be proved (cf. [9]) that for the (almost) Kähler manifolds (pseudo)holomorphic and anti-(pseudo)holomorphic maps $\varphi: M \rightarrow N$ always realize local minima of the energy functional $E(\varphi)$ but, in general, there exist another critical points of $E(\varphi)$, i.e. non-minimal harmonic maps. 
We restrict now to the case, when $M$ is a compact Riemann surface. Denote by $\nabla$ the connection on the bundle $\varphi^{-1}\left(T^{\mathbb{C}} N\right)$ over $M$, induced by the LeviCivita connection ${ }^{N} \nabla$ on the Riemannian manifold $N$. If $z$ is a local complex coordinate on $M$, we set

$$
\delta \varphi=\varphi_{*}(\partial / \partial z), \quad \bar{\delta} \varphi=\varphi_{*}(\partial / \partial \bar{z})
$$

where $\delta \varphi$ and $\bar{\delta} \varphi$ are considered as sections of the bundle $\varphi^{-1}\left(T^{\mathbb{C}} N\right)$. (More generally, we denote by $\delta=\nabla_{\partial / \partial z}, \bar{\delta}=\nabla_{\partial / \partial \bar{z}}$ the components of the connection $\nabla$.) The differential $d \varphi$ is represented in the form

$$
d \varphi=d z \otimes \delta \varphi+d \bar{z} \otimes \bar{\delta} \varphi,
$$

and harmonic map equation (5) may be rewritten in the form

$$
\bar{\delta} \delta \varphi=\left(\nabla_{\partial / \partial \bar{z}} \varphi_{*}\right)\left(\frac{\partial}{\partial z}\right)=0 .
$$

Equivalent formulation

$$
\delta \bar{\delta} \varphi=\left(\nabla_{\partial / \partial z} \varphi_{*}\right)\left(\frac{\partial}{\partial \bar{z}}\right)=0 .
$$

If $N$ is a Kähler manifold, then, according to (6),

$$
\delta \varphi=\partial^{\prime} \varphi+\overline{\partial^{\prime \prime} \varphi}, \quad \bar{\delta} \varphi=\partial^{\prime \prime} \varphi+\overline{\partial^{\prime} \varphi},
$$

and harmonic map equation for $\varphi$ takes on the form

$$
\bar{\delta} \partial^{\prime} \varphi=0
$$

or, equivalently, as

$$
\delta \partial^{\prime \prime} \varphi=0
$$

According to the Koszul-Malgrange theorem (cf. [11]), any complex vector bundle $E$ over a Riemann surface $M$ with a connection $\nabla$ has a unique complex structure $J$, such that $E \rightarrow M$ is a holomorphic vector bundle with respect to $J$, for which the $\bar{\partial}_{J}$-operator coincides with the $(0,1)$-component $\nabla^{0,1}$ of the connection $\nabla$. This complex structure $J$ is called the KM-structure.

In its terms, the harmonicity condition (8) means that $\delta \varphi$ is a holomorphic section of the bundle $\varphi^{-1}\left(T^{\mathbb{C}} N\right)$ with respect to the KM-structure on $\varphi^{-1}\left(T^{\mathbb{C}} N\right)$, induced by the connection ${ }^{N} \nabla$. In the same way, the condition (9) means that $\partial^{\prime} \varphi$ is a holomorphic section of the bundle $\varphi^{-1}\left(T^{1,0} N\right)$. 
2.2. Complex Grassmann manifolds and flag bundles. We are going to apply the twistor approach to the construction of harmonic spheres, presented in Sec. 1.4, to the case when the target manifold $N$ coincides with the complex Grassmannian $G_{r}\left(\mathbb{C}^{d}\right)$. In this case it is natural to choose for the twistor bundle over $G_{r}\left(\mathbb{C}^{d}\right)$ the bundle of complex structures on $G_{r}\left(\mathbb{C}^{d}\right)$, invariant under the action of unitary group $\mathrm{U}(d)$. Such bundles coincide with flag bundles over $G_{r}\left(\mathbb{C}^{d}\right)$ which we define next.

We start by recalling the definition of flag manifolds in $\mathbb{C}^{d}$. For that we fix a decomposition of $d$ into the sum of natural numbers $d=r_{1}+\cdots+r_{n}$ and denote $\mathbf{r}:=\left(r_{1}, \ldots, r_{n}\right)$.

The flag manifold $F_{\mathbf{r}}\left(\mathbb{C}^{d}\right)$ in $\mathbb{C}^{d}$ of type $\mathbf{r}=\left(r_{1}, \ldots, r_{n}\right)$ with $d=r_{1}+\ldots+r_{n}$ consists of flags $\mathcal{W}=\left(W_{1}, \ldots, W_{n}\right)$, i.e. nested sequences of complex subspaces

$$
W_{1} \subset \ldots \subset W_{n}=\mathbb{C}^{d}
$$

such that the dimension of the subspace $E_{1}:=W_{1}$ is equal to $r_{1}$ and dimensions of the subspaces $E_{i}:=W_{i} \ominus W_{i-1}$ are equal to $r_{i}$ for $1<i \leq n$.

In particular, for $\mathbf{r}=(r, d-r)$ the flag manifold

$$
\mathcal{F}_{(r, d-r)}\left(\mathbb{C}^{d}\right)=\left\{\mathcal{E}=\left(E, E^{\perp}\right): \operatorname{dim} E=r\right\}=G_{r}\left(\mathbb{C}^{d}\right)
$$

coincides with the Grassmann manifold of $r$-dimensional subspaces in $\mathbb{C}^{d}$.

We have the following homogeneous representation of the flag manifold

$$
\mathcal{F}_{\mathbf{r}}\left(\mathbb{C}^{d}\right)=\mathrm{U}(d) / \mathrm{U}\left(r_{1}\right) \times \cdots \times \mathrm{U}\left(r_{n}\right) .
$$

There is also another, complex homogeneous representation for this manifold

$$
\mathcal{F}_{\mathbf{r}}\left(\mathbb{C}^{d}\right)=\operatorname{GL}(d, \mathbb{C}) / \mathcal{P}_{\mathbf{r}},
$$

where $\mathcal{P}_{\mathbf{r}}$ is the parabolic subgroup of blockwise upper-triangular matrices of the form

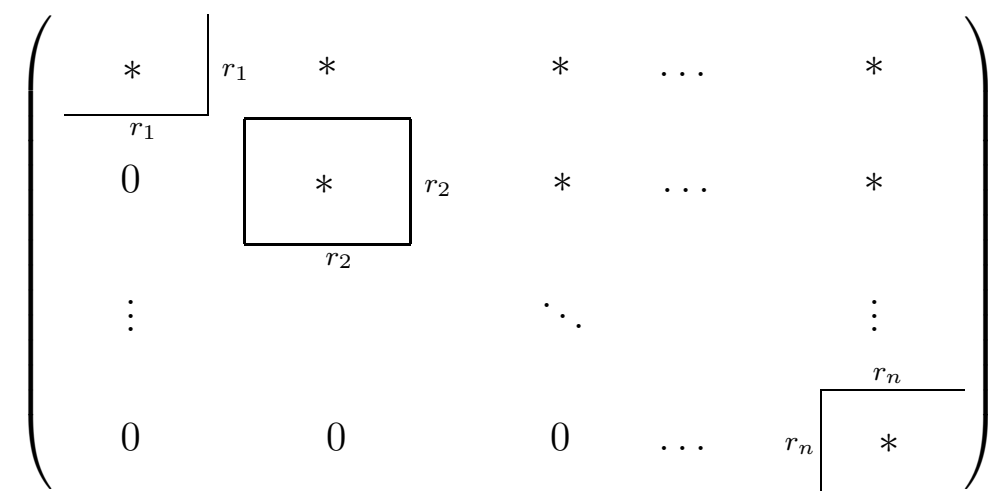

with blocks of dimensions $r_{i} \times r_{i}$ in the boxes.

These representations imply that $\mathcal{F}_{\mathbf{r}}\left(\mathbb{C}^{d}\right)$ has a natural complex structure, which we denote by $J^{1}$. Moreover, $\mathcal{F}_{\mathbf{r}}\left(\mathbb{C}^{d}\right)$, provided with this complex structure, is a compact Kähler manifold. 
In particular case $\mathbf{r}=(r, d-r)$ we obtain the well known homogeneous representations for the Grassmann manifold

$$
G_{r}\left(\mathbb{C}^{d}\right)=\mathrm{U}(d) / \mathrm{U}(r) \times \mathrm{U}(d-r)=\mathrm{GL}(d, \mathbb{C}) / P_{(r, d-r)} .
$$

We construct now a series of homogeneous flag bundles over the Grassmann manifold $G_{r}\left(\mathbb{C}^{c}\right)$. Let $\mathcal{F}=\mathcal{F}_{\mathbf{r}}\left(\mathbb{C}^{N}\right)$ be a flag manifold of type $\mathbf{r}=\left(r_{1}, \ldots, r_{n}\right)$ in $\mathbb{C}^{d}$ with the homogeneous representation

$$
\mathcal{F}=\mathcal{F}_{\mathbf{r}}\left(\mathbb{C}^{N}\right)=\mathrm{U}(d) / \mathrm{U}\left(r_{1}\right) \times \cdots \times \mathrm{U}\left(r_{n}\right) .
$$

On the Lie algebra level this representation corresponds to the decomposition of the complexified Lie algebra $\mathfrak{u}^{\mathbb{C}}(d)$ into the direct orthogonal sum

$$
\begin{aligned}
\mathfrak{u}^{\mathbb{C}}(d) \cong \mathfrak{g l}(d, \mathbb{C}) & \cong \overline{\mathbb{C}^{d}} \otimes \mathbb{C}^{d} \cong\left(\bar{E}_{1} \oplus \cdots \oplus \bar{E}_{n}\right) \otimes\left(E_{1} \oplus \cdots \oplus E_{n}\right) \cong \\
& \cong\left[\mathfrak{u}^{\mathbb{C}}\left(r_{1}\right) \oplus \cdots \oplus \mathfrak{u}^{\mathbb{C}}\left(r_{n}\right)\right] \oplus\left[\bigoplus_{i<j}\left(\bar{E}_{i} E_{j} \oplus \bar{E}_{j} E_{i}\right)\right]
\end{aligned}
$$

(In the latter formula we have omitted the sign of the tensor product in the expression $\bar{E}_{i} E_{j}$ and its conjugate in order to make the formulas more visible. The same rule will be applied in the sequel.)

The above decomposition of the Lie algebra $\mathfrak{u}^{\mathbb{C}}(d)$ implies that the complexified tangent space $T_{o}^{\mathbb{C}} \mathcal{F}$ at the origin $o \in \mathcal{F}$ coincides with

$$
T_{o}^{\mathbb{C}} \mathcal{F}=\bigoplus_{i<j} D_{i j}^{\mathbb{C}}:=\bigoplus_{i<j}\left(\bar{E}_{i} E_{j} \oplus \bar{E}_{j} E_{i}\right)
$$

Every component $D_{i j}$ may be provided with two different complex structures: for one of them its (1,0)-subspace coincides with $\bar{E}_{i} E_{j}$, for another with $\bar{E}_{j} E_{i}$. By the Borel-Hirzebruch theorem [4], any $U(d)$-invariant almost complex structure $J$ on $\mathcal{F}$ is determined by the choice of one of these two complex structures on every $D_{i j}$. The almost complex structure $J^{1}$, for which

$$
T_{o}^{1,0} \mathcal{F}=\bigoplus_{i<j} \bar{E}_{i} E_{j}
$$

is called canonical.

Fix an ordered subset $\sigma \subset\{1, \ldots, n\}$ and set $r:=\sum_{i \in \sigma} r_{i}$. We can associate with any such subset $\sigma$ the homogeneous bundle

$$
\pi_{\sigma}: \quad \mathcal{F}_{\mathbf{r}}\left(\mathbb{C}^{N}\right)=\frac{U(d)}{U\left(r_{1}\right) \times \ldots \times U\left(r_{n}\right)} \longrightarrow \frac{U(d)}{U(r) \times U(d-r)}=G_{r}\left(\mathbb{C}^{d}\right)
$$

by mapping $\left(E_{1}, \ldots, E_{n}\right) \longmapsto E=\bigoplus_{i \in \sigma} E_{i}$.

The complexified tangent bundle $T^{\mathbb{C}} \mathcal{F}_{\mathbf{r}}\left(\mathbb{C}^{N}\right)$ is decomposed into the direct sum of vertical and horizontal subbundles. Namely, the vertical subspace at the origin coincides with $\bigoplus_{i, j} D_{i j}^{\mathbb{C}}$, where $i<j$ and either $i, j \in \sigma$, or $i, j \notin \sigma$. 
Respectively, the horizontal subspace at the origin is equal to $\bigoplus_{i, j} D_{i j}^{\mathbb{C}}$, where $i<j$ and either $i \in \sigma, j \notin \sigma$, or $i \notin \sigma, j \in \sigma$.

We introduce, along with the canonical complex structure $\mathcal{J}^{1}$, a new $\mathrm{U}(d)$ invariant almost complex structure $\mathcal{J}_{\sigma}^{2}$ on $\mathcal{F}_{\mathbf{r}}\left(\mathbb{C}^{N}\right)$, by setting it equal to $\mathcal{J}_{\sigma}^{2}=$ $\mathcal{J}^{1}$ on horizontal tangent vectors and $\mathcal{J}_{\sigma}^{2}=-\mathcal{J}^{1}$ on vertical tangent vectors.

With this almost complex structure on $\mathcal{F}_{\mathbf{r}}\left(\mathbb{C}^{N}\right)$ we have the following analogue of the Eells-Salamon theorem from Sec. 1.4.

Theorem 2 (Burstall-Salamon). The flag bundle

$$
\pi_{\sigma}:\left(F_{\mathbf{r}}\left(\mathbb{C}^{d}\right), \mathcal{J}_{\sigma}^{2}\right) \longrightarrow G_{r}\left(\mathbb{C}^{d}\right),
$$

provided with the almost complex structure $\mathcal{J}_{\sigma}^{2}$, is a twistor bundle, i.e. the projection $\varphi=\pi_{\sigma} \circ \psi$ of any holomorphic map $\psi: M \rightarrow F_{\mathbf{r}}\left(\mathbb{C}^{d}\right)$ from the Riemann surface $M$ to $G_{r}\left(\mathbb{C}^{d}\right)$ is a harmonic map $\varphi: M \rightarrow G_{r}\left(\mathbb{C}^{d}\right)$. In the case when $M=\mathbb{P}^{1}$ is the Riemann sphere, the converse of this assertion is also true: any harmonic sphere $\varphi: \mathbb{P}^{1} \rightarrow G_{r}\left(\mathbb{C}^{d}\right)$ in $G_{r}\left(\mathbb{C}^{d}\right)$ may be obtained as the projection of a holomorphic sphere in some flag bundle $\pi_{\sigma}: F_{\mathbf{r}}\left(\mathbb{C}^{d}\right) \rightarrow G_{r}\left(\mathbb{C}^{d}\right)$.

In Section 3.2 we shall generalize this theorem to harmonic maps with values in Hilbert-Schmidt Grassmannians.

\section{Harmonic maps into Hilbert-Schmidt Grassmanninans}

In this Section we prove our main result - an infinite-dimensional generalization of the Burstall-Salamon theorem from Section 2.2.

3.1. Hilbert-Schmidt Grassmannians. Consider a complex Hilbert space $H$, provided with a polarization. It means that $H$ is decomposed

$$
H=H_{+} \oplus H_{-}
$$

into the direct orthogonal sum of closed infinite-dimensional subspaces $H_{ \pm}$. For example, in the case of the Hilbert space $H=L_{0}^{2}\left(S^{1}, \mathbb{C}\right)$ of square integrable functions on $S^{1}$ with zero average one can take for such subspaces

$$
H_{ \pm}=\left\{\gamma \in H: \gamma=\sum_{ \pm k>0} \gamma_{k} e^{i k \theta}\right\}
$$

Any bounded linear operator $A \in L(H)$ with respect to the polarization (12) can be written in the block form

$$
A=\left(\begin{array}{ll}
a & b \\
c & d
\end{array}\right)=\left(\begin{array}{ll}
a: H_{+} \rightarrow H_{+}, & b: H_{-} \rightarrow H_{+} \\
c: H_{+} \rightarrow H_{-}, & d: H_{-} \rightarrow H_{-}
\end{array}\right) .
$$

Denote by $\mathrm{GL}(H)$ the group of linear bounded operators in $H$, having a bounded inverse, and introduce the Hilbert-Schmidt group $\mathrm{GL}_{\mathrm{HS}}(H)$, consisting of operators $A \in \mathrm{GL}(H)$, for which the "off-diagonal" terms $b$ and $c$ are 
Hilbert-Schmidt operators. In other words, the group $\mathrm{GL}_{\mathrm{HS}}(H)$ consists of operators $A \in \mathrm{GL}(H)$, for which the "off-diagonal" terms $b$ and $c$ are "small" with respect to the "diagonal" terms $a$ and $d$. We denote also by $\mathrm{U}_{\mathrm{HS}}(H)$ the intersection of $\mathrm{GL}_{\mathrm{HS}}(H)$ with the group $\mathrm{U}(H)$ of unitary operators in $H$.

As in finite-dimensional situation, there is a Grassmann manifold $\operatorname{Gr}_{\mathrm{HS}}(H)$, called the Hilbert-Schmidt Grassmannian, related to the group $\mathrm{GL}_{\mathrm{HS}}(H)$.

The Hilbert-Schmidt Grassmannian $\operatorname{Gr}_{\mathrm{HS}}(H)$ consists of all closed subspaces $W \subset H$ such that the orthogonal projection $\mathrm{pr}_{+}: W \rightarrow H_{+}$is a Fredholm operator, while the orthogonal projection $\mathrm{pr}_{-}: W \rightarrow H_{-}$is a Hilbert-Schmidt operator. Equivalently: a subspace $W \in \operatorname{Gr}_{\mathrm{HS}}(H)$ iff it coincides with the image of a linear operator $w: H_{+} \rightarrow H$ such that $w_{+}:=\operatorname{pr}_{+} \circ w$ is a Fredholm operator, and $w_{-}:=\mathrm{pr}_{-} \circ w$ is a Hilbert-Schmidt operator.

In other words, $\operatorname{Gr}_{\mathrm{HS}}(H)$ consists of the subspaces $W \subset H$, which differ "little" from the subspace $H_{+}$in the sense that projection $\mathrm{pr}_{+}: W \rightarrow H_{+}$is "close" to an isomorphism and projection $\mathrm{pr}_{-}: W \rightarrow H_{-}$is "small".

We have the following homogeneous space representation of $\operatorname{Gr}_{\mathrm{HS}}(H)$ :

$$
\operatorname{Gr}_{\mathrm{HS}}(H)=\mathrm{U}_{\mathrm{HS}}(H) / \mathrm{U}\left(H_{+}\right) \times \mathrm{U}\left(H_{-}\right) .
$$

Since $\mathrm{U}_{\mathrm{HS}}(H)$ acts transitively on the Grassmannian $\operatorname{Gr}_{\mathrm{HS}}(H)$, we can construct an $\mathrm{U}_{\mathrm{HS}}(H)$-invariant Kähler metric on $\mathrm{Gr}_{\mathrm{HS}}(H)$ from an inner product on the tangent space $T_{H_{+}} \operatorname{Gr}_{\mathrm{HS}}(H)$ at the origin $H_{+} \in \operatorname{Gr}_{\mathrm{HS}}(H)$, invariant under the action of the isotropy subgroup $\mathrm{U}\left(H_{+}\right) \times \mathrm{U}\left(H_{-}\right)$. The tangent space $T_{H_{+}} \mathrm{Gr}_{\mathrm{HS}}(H)$ coincides with the space of Hilbert-Schmidt operators $\operatorname{HS}\left(H_{+}, H_{-}\right)$and invariant inner product on it is given by the formula

$$
(A, B) \longmapsto \operatorname{Re}\left\{\operatorname{tr}\left(A B^{\dagger}\right)\right\}, \quad A, B \in \operatorname{HS}\left(H_{+}, H_{-}\right) .
$$

Note that the imaginary part of the complex inner product $\operatorname{tr}\left(A B^{\dagger}\right)$ determines a non-degenerate invariant 2-form on $T_{H_{+}} \operatorname{Gr}_{\mathrm{HS}}(H)$, which extends to an $\mathrm{U}_{\mathrm{HS}}(H)$-invariant symplectic structure on the manifold $\operatorname{Gr}_{\mathrm{HS}}(H)$. Hence, we have a Kähler structure on $\operatorname{Gr}_{\mathrm{HS}}(H)$, which makes it a Kähler Hilbert manifold.

There is an evident obstacle to the immediate extension of the results, obtained in Section 2.2 for finite-dimensional Grassmannians, to $\operatorname{Gr}_{\mathrm{HS}}(H)$. Namely, all subspaces $W \in \operatorname{Gr}_{\mathrm{HS}}(H)$ are infinite-dimensional, i.e. in some sense have the same infinite "dimension" and the problem is how to compare them. Fortunately, there is a replacement of the notion of dimension in Hilbert-Schmidt Grassmannian, called the "virtual dimension", which can be used for the comparison of different subspaces.

In more detail, $\operatorname{Gr}_{\mathrm{HS}}(H)$ has a countable number of connected components, numerated by the index of the Fredholm operator $w_{+}$for a subspace $W \in$ $\operatorname{Gr}_{\mathrm{HS}}(H)$. We say that $W$ has the virtual dimension $r$ if the index of $w_{+}$ 
is equal to $r$. Denote by $\mathrm{G}_{r}(H)$ the component of $\operatorname{Gr}_{\mathrm{HS}}(H)$, consisting of subspaces $W$ of virtual dimension $r$.

Then we have the following decomposition of $\operatorname{Gr}_{\mathrm{HS}}(H)$ into the disjoint union of its connected components $\mathrm{G}_{r}(H)$ :

$$
\operatorname{Gr}_{\mathrm{HS}}(H)=\bigcup_{r} \mathrm{G}_{r}(H) \text {. }
$$

Due to this decomposition, the study of harmonic maps of Riemann surfaces into $\operatorname{Gr}_{\mathrm{HS}}(H)$ is reduced to the study of harmonic maps into Grassmannians $\mathrm{G}_{r}(H)$ of virtual dimension $r$, which may be carried on along the same lines, as in the case of Grassmann manifold $G_{r}\left(\mathbb{C}^{d}\right)$.

3.2. Harmonic maps into Hilbert-Schmidt Grassmannians. Let $M$ be a Riemann surface. Denote by $M \times H$ the trivial bundle $M \times H \rightarrow M$ where $H$ is a complex Hilbert space provided with the polarization (12). We shall consider the subbundles $E \subset M \times H$, having the fibres $E_{p} \in \operatorname{Gr}_{\mathrm{HS}}(H)$ for $p \in$ $M$. Any bundle $E$ of this type defines a map $\varphi_{E}: M \longrightarrow \operatorname{Gr}_{\mathrm{HS}}(H)$ by setting: $\varphi_{E}(p):=$ the fibre $E_{p}$ at $p \in M$. Conversely, any map $\varphi: M \rightarrow \operatorname{Gr}_{\mathrm{HS}}(H)$ defines a subbundle $E \subset M \times \operatorname{Gr}_{\mathrm{HS}}(H)$.

Consider a smooth map of a Riemann surface $M$ into $\operatorname{Grassmannian} \operatorname{Gr}_{\mathrm{HS}}(H)$. Denote by $\pi$ and $\pi^{\perp}$ the orthogonal projections of $M \times H$ onto the subbundle $E$ and its orthogonal complement $E^{\perp}$ respectively. The bundle $E$ is provided with the complex KM-structure which is determined in a local chart on $M$ with local coordinate $z$ by the $\bar{\partial}$-operator

$$
\partial_{E}^{\prime \prime}=\pi \circ \frac{\partial}{\partial \bar{z}} \circ \pi
$$

The inverse image $\varphi_{E}^{-1}\left(T^{\mathbb{C}} \operatorname{Gr}_{\mathrm{HS}}(H)\right)$ of the complexified tangent bundle of $\operatorname{Gr}_{\mathrm{HS}}(H)$ under the map $\varphi_{E}$ admits the decomposition

$$
\varphi_{E}^{-1}\left(T^{\mathbb{C}} \operatorname{Gr}_{\mathrm{HS}}(H)\right) \cong \bar{E} E^{\perp} \oplus \overline{E^{\perp}} E .
$$

In terms of this decomposition the differential of $\varphi_{E}$ has local components

$$
A_{E}^{\prime}:=\pi^{\perp} \circ \frac{\partial}{\partial z} \circ \pi, \quad A_{E}^{\prime \prime}:=\pi^{\perp} \circ \frac{\partial}{\partial \bar{z}} \circ \pi .
$$

(In the sequel we sometimes omit the sign $\circ$ to simplify the formulas.) In particular, the bundle $E \subset M \times H$ is holomorphic $\Longleftrightarrow A_{E}^{\prime \prime}=0$, and in this case the KM-structure on $E$ coincides with the complex structure, induced from $M \times H$. Then

$$
\begin{aligned}
0=\pi^{\perp}\left[\frac{\partial}{\partial z}(\pi+\right. & \left.\left.\pi^{\perp}\right) \frac{\partial}{\partial \bar{z}}-\frac{\partial}{\partial \bar{z}}\left(\pi+\pi^{\perp}\right) \frac{\partial}{\partial z}\right] \pi= \\
& =A_{E}^{\prime} \partial_{E}^{\prime \prime}+\partial_{E^{\perp}}^{\prime} A_{E}^{\prime \prime}-A_{E}^{\prime \prime} \partial_{E}^{\prime}-\partial_{E^{\perp}}^{\prime \prime} A_{E}^{\prime}=A_{E}^{\prime} \partial_{E}^{\prime \prime}-\partial_{E^{\perp}}^{\prime \prime} A_{E}^{\prime} .
\end{aligned}
$$


In other words, $A_{E}^{\prime} \in \operatorname{Hom}\left(E, E^{\perp}\right)$ is holomorphic with respect to the KMstructures on $E$ and $E^{\perp}$.

In general, we call a bundle $E \subset M \times H$ harmonic if

$$
A_{E}^{\prime} \circ \partial_{E}^{\prime \prime}=\partial_{E^{\perp}}^{\prime \prime} \circ A_{E}^{\prime} \text {. }
$$

The harmonicity of $E$ is equivalent to the harmonicity of the map $\varphi_{E}: M \rightarrow$ $G_{r}(H)$ (cf. [6]). Note also that $E$ is harmonic $\Longleftrightarrow$ its orthogonal complement $E^{\perp}$ is harmonic.

Now we generalize this situation to the maps into infinite-dimensional flag manifolds.

3.3. Holomorphic maps into Hilbert-Schmidt flag manifolds. Introduce first the Hilbert-Schmidt flag manifolds. For that fix an $n$-tuple $\mathbf{r}=$ $\left(r_{1}, \ldots, r_{n}\right)$ of integers. The Hilbert-Schmidt flag manifold $F_{\mathbf{r}}(H)$ consists of the flags of the form

$$
\mathcal{E} \equiv\left(E_{1}, \ldots E_{n}\right)
$$

where $E_{k} \equiv W_{\text {in }}, E_{l} \equiv W_{\text {out }}$ are closed infinite-dimensional subspaces in $H$ and

$$
E_{1}, \ldots, E_{k-1}, E_{k+1}, \ldots, E_{l-1}, E_{l+1}, \ldots, E_{n}
$$

are finite-dimensional subspaces having the following properties:

(1) the projection $\mathrm{pr}_{+}: W_{\mathrm{in}} \rightarrow H_{+}$is a Fredholm operator of index $r_{k}$ while the projection $\mathrm{pr}_{-}: W_{\text {in }} \rightarrow H_{-}$is a Hilbert-Schmidt operator;

(2) the projection $\mathrm{pr}_{-}: W_{\text {out }} \rightarrow H_{-}$is a Fredholm operator of index $r_{l}$ while the projection $\mathrm{pr}_{+}: W_{\text {out }} \rightarrow H_{+}$is a Hilbert-Schmidt operator;

(3) $E_{i}$ with $i=1, \ldots, k-1, k+1, \ldots, l-1, l+1, \ldots, n$ are $r_{i}$-dimensional vector subspaces in $H$;

(4) all subspaces $E_{i}$ with $i=1, \ldots, n$ are pairwise orthogonal and their direct sum is equal to $H: E_{1} \oplus \ldots \oplus E_{n}=H$.

To simplify the notation, we say that $\mathcal{E}=\left(E_{1}, \ldots E_{n}\right)$ is a virtual flag of virtual dimension $\mathbf{r}=\left(r_{1}, \ldots, r_{n}\right)$ having in mind that $r_{k}$ (resp. $\left.r_{l}\right)$ are integers, equal to the virtual dimension of $E_{k}=W_{\text {in }}$ (resp. $\left.E_{l}=W_{\text {out }}\right)$, while other $r_{i}$ 's are positive integers, equal to the dimensions of $E_{i}$ 's for $i \neq k, l$.

The tangent space to $F_{\mathbf{r}}(H)$ at the origin, as in the finite-dimensional case, is the direct sum of four different terms

$$
\begin{array}{r}
T^{\mathbb{C}}\left(F_{\mathbf{r}}(H)\right) \cong \bigoplus_{1 \leq i, j \neq k, l, i<j \leq n}\left[\bar{E}_{i} E_{j} \oplus \bar{E}_{j} E_{i}\right] \oplus \bigoplus_{1 \leq i \leq n, i \neq k}\left[\bar{W}_{\text {in }} E_{i} \oplus \bar{E}_{i} W_{\text {in }}\right] \\
\oplus \bigoplus_{1 \leq i \leq n, i \neq l}\left[\bar{W}_{\text {out }} E_{i} \oplus \bar{E}_{i} W_{\text {out }}\right] \oplus\left[\bar{W}_{\text {in }} W_{\text {out }} \oplus \bar{W}_{\text {out }} W_{\text {in }}\right] .
\end{array}
$$

The tangent space to $\left.\operatorname{Gr}_{\mathrm{HS}}(H)\right)$ looks the same if we set $E_{i}=0$ for all $E_{i}$ 's except for $i=k, l$. 
Generalizing the situation, studied in Sec. 3.2, let us consider an arbitrary collection $\mathcal{E}=\left(E_{1}, \ldots, E_{n}\right)$ of mutually orthogonal subbundles $E_{i}$ in $M \times H$ of virtual dimension $\mathbf{r}=\left(r_{1}, \ldots, r_{n}\right)$, generating the decomposition of $M \times H$ into the direct orthogonal sum

$$
M \times H=\bigoplus_{i=1}^{n} E_{i} .
$$

We call such a collection of subbundles $\mathcal{E}=\left(E_{1}, \ldots, E_{n}\right)$ the moving flag on $M$. It determines, in the same way as before, a map $\psi_{\mathcal{E}}: M \rightarrow F_{\mathbf{r}}(H) \equiv \mathcal{F}$ by assigning to a point $p \in M$ the flag, defined by the subspaces $\left(E_{1, p}, \ldots, E_{n, p}\right)$. Conversely, any smooth map $\psi: M \rightarrow \mathcal{F}$ determines a moving flag $\mathcal{E}=$ $\left(E_{1}, \ldots, E_{n}\right)$, where $E_{i}=\psi^{-1} T_{i}$ is the pull-back of a natural tautological bundle $T_{i} \rightarrow F_{\mathbf{r}}(H)$ : the fibre of $T_{i}$ at $\mathcal{E} \in \mathcal{F}$ coincides, by definition, with the subspace $E_{i}$ for $1 \leq i \leq n$.

As in the Grassmann case, the differential $\psi_{\mathcal{E}}$ is determined locally by the components

$$
A_{i j}^{\prime}=\pi_{i} \circ \frac{\partial}{\partial z} \circ \pi_{j}, \quad A_{i j}^{\prime \prime}=\pi_{i} \circ \frac{\partial}{\partial \bar{z}} \circ \pi_{j},
$$

where $\pi_{i}: M \times H \rightarrow E_{i}$ is the orthogonal projection. Note that by construction $A_{i j}^{\prime \prime}=-\left(A_{j i}^{\prime}\right)^{*}$.

Each of the subbundles $E_{i}$ of the trivial bundle $M \times H$ is provided with the KM-structure which coincide with the complex structure, induced from $M \times$ $H$. Also the components $A_{i j}^{\prime}, A_{i j}^{\prime \prime}$ satisfy the harmonicity and holomorphicity conditions similar to those in Sec. 3.2.

We introduce now an almost complex structure on the Hilbert-Schmidt flag bundle $F_{\mathbf{r}}(H)$, analogous to the almost complex structure $\mathcal{J}_{\sigma}^{2}$ from Sec. 2.2, As in the Grassmannian case, an almost complex structure on $F_{\mathbf{r}}(H)$ is fixed by choosing the $(1,0)$-component in each of the summands of

$$
T^{\mathbb{C}}\left(F_{\mathbf{r}}(H)\right) \cong \bigoplus_{1 \leq i<j \leq n}\left[\bar{E}_{i} E_{j} \oplus \bar{E}_{j} E_{i}\right]
$$

(We recall that (14) is just a concise form of (??).)

To define the almost complex structure $\mathcal{J}_{\sigma}^{2}$, we fix an ordered subset $\sigma \subset$ $\{1, \ldots, n\}$. Then for the associated almost complex structure $\mathcal{J}_{\sigma}^{2}$ we choose for $i, j \in\{1, \ldots, n\}, i<j$, the (1,0)-component in $(i, j)$-summand in (14), equal to $\bar{E}_{j} E_{i}$ if $i, j \in \sigma$ or $i, j \notin \sigma$, and to $\bar{E}_{i} E_{j}$ if $i \in \sigma, j \notin \sigma$ or $i \notin \sigma, j \in \sigma$.

3.4. Twistor bundle over the Hilbert-Schmidt Grassmannian. We construct now the Hilbert-Schmidt flag bundle over the Grassmannian. Suppose that $\sigma$ is a fixed ordered subset in $\{1, \ldots, n\}$ and set $r=\sum_{i \in \sigma} r_{i}$. Then 
we define the Hilbert-Schmidt flag bundle

$$
\pi_{\sigma}: F_{\mathbf{r}}(H) \longrightarrow G_{r}(H)
$$

by mapping

$$
\mathcal{E}=\left(E_{1}, \ldots, E_{n}\right) \longmapsto E:=\bigoplus_{i \in \sigma} E_{i} .
$$

With this definition we can prove the following

Theorem 3. Let $\sigma$ be an ordered subset in $\{1, \ldots, n\}$ such that $k \in \sigma, l \notin \sigma$. Then the map $\pi_{\sigma}$ of the Hilbert-Schmidt flag manifold $F_{\mathbf{r}}(H)$, provided with the almost complex structure $\mathcal{J}_{\sigma}^{2}$ to $G_{r}(H)$ :

$$
\pi_{\sigma}: F_{\mathbf{r}}(H) \longrightarrow G_{r}(H)
$$

is a twistor bundle. It means that for any $\mathcal{J}_{\sigma}^{2}$-holomorphic map $\psi: M \rightarrow$ $F_{\mathbf{r}}(H)$ its projection $\varphi=\pi_{\sigma} \circ \psi: M \rightarrow G_{r}(H)$ is harmonic.

Proof. To prove the theorem, it is sufficient to show that for any moving flag $\mathcal{E}=\left(E_{1}, \ldots, E_{n}\right)$, corresponding to a $\mathcal{J}_{\sigma}^{2}$-holomorphic map $\psi_{\mathcal{E}}: M \rightarrow \mathcal{F}$, the bundle $E:=\bigoplus_{i \in \sigma} E_{i}$ is harmonic. The holomorphicity of the map $\psi_{\mathcal{E}}$ means that

$$
A_{i j}^{\prime}=0=A_{j i}^{\prime \prime} \quad \text { if } \begin{cases}i>j & \text { and } \quad i, j \in \sigma \text { or } i, j \notin \sigma, \\ i<j & \text { and } \quad i \in \sigma, j \notin \sigma \text { or } i \notin \sigma, j \in \sigma .\end{cases}
$$

We have to prove that the bundle $E$, i.e. that

$$
A_{E}^{\prime} \circ \partial_{E}^{\prime \prime}=\partial_{E \perp}^{\prime \prime} \circ A_{E}^{\prime}
$$

Take $s<t$ with $s \in \sigma, t \notin \sigma$. Then

$$
\begin{array}{r}
0=\pi_{t} \sum_{i}\left[\frac{\partial}{\partial \bar{z}} \pi_{i} \frac{\partial}{\partial z}-\frac{\partial}{\partial z} \pi_{i} \frac{\partial}{\partial \bar{z}}\right] \pi_{s}=\sum_{i}\left(A_{t i}^{\prime \prime} A_{i s}^{\prime}-A_{t i}^{\prime} A_{i s}^{\prime \prime}\right)= \\
\sum_{i \notin \sigma} A_{t i}^{\prime \prime} A_{i s}^{\prime}-\sum_{i \in \sigma} A_{t i}^{\prime} A_{i s}^{\prime \prime}=\left(\sum_{i \notin \sigma} A_{t i}^{\prime \prime}\right)\left(\sum_{i \notin \sigma} A_{i s}^{\prime}\right)-\left(\sum_{i \in \sigma} A_{t i}^{\prime}\right)\left(\sum_{i \in \sigma} A_{i s}^{\prime \prime}\right) \\
=\pi_{t}\left(\partial_{E \perp}^{\prime \prime} \circ A_{E}^{\prime}-A_{E}^{\prime} \circ \partial_{E}^{\prime \prime}\right) \pi_{s} .
\end{array}
$$

Analogous relations are satisfied for $s>t$, which implies that $A_{E}^{\prime} \circ \partial_{E}^{\prime \prime}=$ $\partial_{E^{\perp}}^{\prime \prime} \circ A_{E}^{\prime}$, i.e. $E$ is harmonic.

In the case when $M$ is the Riemann sphere $\mathbb{P}^{1}$, it is possible to prove a conversion of the theorem [3, based on an infinite-dimensional extension of Birkhoff-Grothendieck classification theorem for holomorphic vector bundles over $\mathbb{P}^{1}$, considered in the next Section 3.5 . 


\subsection{Infinite-dimensional version of the Birkhoff-Grothedieck theo-}

rem. Recall that the standard Birkhoff-Grothendieck theorem asserts that any holomorphic vector bundle $E$ of rank $d$ over $\mathbb{P}^{1}$ is equivalent to the direct sum of holomorphic line bundles $\mathcal{O}\left(\kappa_{1}\right) \oplus \ldots \oplus \mathcal{O}\left(\kappa_{d}\right)$ with some integers $\kappa_{1} \geq \ldots \geq \kappa_{d}$.

In terms of transition functions a holomorphic vector bundle $E$ over $\mathbb{P}^{1}$ is determined by an invertible holomorphic $d \times d$-matrix function $f$, defined in a neighbourhood $U_{0} \cap U_{\infty}$ of the equator of the Riemann sphere $\mathbb{P}^{1}$. Here the sets $U_{0}:=\mathbb{P}^{1} \backslash\{\infty\}$ and $U_{\infty}:=\mathbb{P}^{1} \backslash\{0\}$ form the standard open covering of $\mathbb{P}^{1}$. In these terms the Birkhoff-Grothendieck theorem is equivalent to the factorization of the transition function $f$ in the form

$$
f(z)=f_{0}(z) d(z) f_{\infty}(z)
$$

where $f_{0}$ (resp. $f_{\infty}$ ) is a holomorphic invertible matrix function in $U_{0}$ (resp. $\left.U_{\infty}\right)$ and $d(z)$ is the diagonal matrix function of the form

$$
d(z)=\operatorname{diag}\left(z^{\kappa_{1}}, \ldots, z^{\kappa_{d}}\right) .
$$

There exists still another formulation of Birkhoff-Grothendieck theorem in the form of Harder-Narasimhan filtration. Suppose that $E$ is a holomorphic vector bundle $E$ of rank $d$ over $\mathbb{P}^{1}$, identified with a subbundle of the trivial rank $d$ bundle over $\mathbb{P}^{1}$. Then there exists a filtration of $E$ by holomorphic subbundles

$$
0=\mathcal{B}_{0} \subset \mathcal{B}_{1} \subset \ldots \subset \mathcal{B}_{k}=E,
$$

having the quotients of the form

$$
\mathcal{B}_{i} / \mathcal{B}_{i-1} \cong \underbrace{L^{\beta_{i}} \oplus \ldots \oplus L^{\beta_{i}}}_{b_{i} \text { times }},
$$

where $L^{\beta_{i}}$ is the $\beta_{i}$-th power of the tautological line bundle $L$ over $\mathbb{P}^{1}$ and $\beta_{1}>\cdots>\beta_{k}$. The subbundle $\mathcal{B}_{i}$ can be defined as the smallest holomorphic subbundle of $E$, containing the images of all meromorphic sections of $E$ with divisors of degree, greater or equal to $\beta_{i}$. Using the Hermitian metric on $\mathbb{C}^{d}$, we can identify the quotient $\mathcal{B}_{i} / \mathcal{B}_{i-1}$ with the orthogonal complement $B_{i}$ of $\mathcal{B}_{i-1}$ in $\mathcal{B}_{i}$

In the infinite-dimensional setting the Birkhoff-Grothendieck theorem, proved in [13], [14, implies that any holomorphic Hilbert-space bundle $E$ over $\mathbb{P}^{1}$ with the structure group $\mathrm{GL}_{\mathrm{HS}}(H)$, consisting of invertible operators of the form $I+T$ is equivalent to the direct sum of a finite number of holomorphic line bundles and a trivial Hilbert-space bundle.

In terms of transition functions a holomorphic Hilbert-space bundle $E$ is determined by a holomorphic operator function $F(z)=I+T(z)$ with values in the group $\operatorname{GL}_{\mathrm{HS}}(H)$, defined in the neighborhood $U_{0} \cap U_{\infty}$. In these terms 
the Birkhoff-Grothendieck theorem is equivalent to the factorization of the transition function $F$ in the form

$$
F(z)=F_{0}(z) D(z) F_{\infty}(z)
$$

where $F_{0}(z)=I+T_{0}(z)$ (resp. $\left.F_{\infty}(z)=I+T_{\infty}(z)\right)$ is a holomorphic operator function in $U_{0}$ (resp. $U_{\infty}$ ) with values in $\operatorname{GL}_{\mathrm{HS}}(H)$ and $D(z)$ is the diagonal operator function of the form

$$
D(z)=\operatorname{diag}\left(z^{\kappa_{1}}, z^{\kappa_{2}}, \ldots\right)
$$

where $\kappa_{1} \geq \kappa_{2} \geq \ldots$ are some integers such that all of them, apart from a finite number, are equal to 0 .

In terms of Harder-Narasimhan filtration this theorem means that there exists a filtration of $E$ by holomorphic subbundles

$$
0=\mathcal{B}_{0} \subset \mathcal{B}_{1} \subset \ldots \subset \mathcal{B}_{s}=E,
$$

having the quotients of the form

$$
\mathcal{B}_{i} / \mathcal{B}_{i-1} \cong \underbrace{L^{\beta_{i}} \oplus \ldots \oplus L^{\beta_{i}}}_{b_{i} \text { times }} \cong b_{i} L^{\beta_{i}}
$$

for $i=1, \ldots, s, i \neq k$, and

$$
\mathcal{B}_{k} / \mathcal{B}_{k-1} \cong E_{k}=W_{\text {in }}
$$

where $\beta_{1}>\cdots>\beta_{s}$. Using the Hermitian metric on $H$, we can identify the quotient $\mathcal{B}_{i} / \mathcal{B}_{i-1}$ with the orthogonal complement $B_{i}$ of $\mathcal{B}_{i-1}$ in $\mathcal{B}_{i}$. Note that the induced complex structure on $\mathcal{B}_{i} / \mathcal{B}_{i-1}$ coincides with the complex KM-structure on $B_{i}$.

\subsection{Twistor description of harmonic spheres in Hilbert-Schmidt Grass-}

mannians. Now we are ready to formulate the converse of theorem 3 .

Theorem 4. Let $\varphi: \mathbb{P}^{1} \rightarrow G_{r}(H)$ be a harmonic map. Then there exist a Hilbert-Schmidt flag bundle

$$
\pi_{\sigma}: F_{\mathbf{r}}(H) \longrightarrow G_{r}(H)
$$

and a $\mathcal{J}_{\sigma}^{2}$-holomorphic map $\psi: \mathbb{P}^{1} \rightarrow F_{\mathbf{r}}(H)$ such that $\varphi$ coincides with the projection $\pi_{\sigma} \circ \psi$ of the map $\psi$.

Proof. The proof of this theorem goes on along the same lines as in the finitedimensional case (cf. [5]).

Associate with the harmonic map $\varphi: \mathbb{P}^{1} \rightarrow G_{r}(H)$ the bundle $E$ over $\mathbb{P}^{1}$ and provide it with the complex KM-structure. With respect to this complex 
structure $E$ becomes a holomorphic Hilbert-space bundle. So by the BirkhoffGrothendieck theorem (in Harder-Narasimhan form) there exists a filtration of $E$ by holomorphic subbundles

$$
0=\mathcal{B}_{0} \subset \mathcal{B}_{1} \subset \ldots \subset \mathcal{B}_{s}=E,
$$

with the quotients of the form

$$
\mathcal{B}_{i} / \mathcal{B}_{i-1} \cong b_{i} L^{\beta_{i}}
$$

for $i=1, \ldots, s, i \neq k$, and $\mathcal{B}_{k} / \mathcal{B}_{k-1} \cong E_{k}=W_{\mathrm{in}}$, such that $\beta_{1}>\cdots>\beta_{s}$ (where we set $\beta_{k}=0, b_{k}=1$ ).

We can construct an analogous filtration for the orthogonal complement $E^{\perp}$ of $E$ in $\mathbb{P}^{1} \times H \rightarrow \mathbb{P}^{1}$ :

$$
0=\mathcal{C}_{0} \subset \mathcal{C}_{1} \subset \ldots \subset \mathcal{C}_{t}=E^{\perp}
$$

with quotients of the form

$$
\mathcal{C}_{i} / \mathcal{C}_{i-1} \cong c_{i} L^{\gamma_{i}}
$$

for $i=1, \ldots, t, i \neq l$, and

$$
\mathcal{C}_{l} / \mathcal{C}_{l-1} \cong E_{l}=W_{\text {out }}
$$

such that $\gamma_{1}>\cdots>\gamma_{t}$ (where we also set $\gamma_{l}=0, c_{l}=1$ ). We identify again the quotient $\mathcal{C}_{i} / \mathcal{C}_{i-1}$ with the orthogonal complement $C_{i}$ of $\mathcal{C}_{i-1}$ in $\mathcal{C}_{i}$.

We collect now the subbundles $B_{1}, \ldots, B_{s}, C_{1}, \ldots, C_{t}$ into a single collection of $n=s+t$ subbundles, denoted by $E_{1}, \ldots, E_{n}$, so that each of $E_{i}$ is isomorphic to the direct sum of $e_{i}$ copies of $L^{\delta_{i}}$ and $\delta_{1} \leq \cdots \leq \delta_{n}$. (If for some $j$ we have $\delta_{j}=\delta_{j+1}$, we arrange the associated subbundles $E_{j}, E_{j+1}$ in such a way that $E_{j}$ corresponds to some $B_{p}$ and $E_{j+1}$ to some $C_{q}$.) We introduce the subset $\sigma \subset\{1,2, \ldots, n\}$, uniquely defined by the equalities

$$
E=\bigoplus_{i \in \sigma} E_{i}, \quad E^{\perp}=\bigoplus_{i \notin \sigma} E_{i} .
$$

Denote by $\mathcal{E}:=\left(E_{1}, \ldots, E_{n}\right)$ the moving flag, determined by the subbundles $E_{1}, \ldots, E_{n}$, and by $\psi_{\mathcal{E}}: \mathbb{P}^{1} \rightarrow F_{\mathbf{r}}(H)$ the corresponding map, associated with $\mathcal{E}$. We have to prove that this map is $\mathcal{J}_{\sigma}^{2}$-holomorphic. In other words, we should prove that

$$
A_{i j}^{\prime}=0=A_{j i}^{\prime \prime} \quad \text { if } \begin{cases}i>j & \text { and } \quad i, j \in \sigma \text { or } i, j \notin \sigma, \\ i<j & \text { and } \quad i \in \sigma, j \notin \sigma \text { or } i \notin \sigma, j \in \sigma .\end{cases}
$$

Suppose first that $i>j$ and $i, j \in \sigma$. Then $\delta_{i}>\delta_{j}$ and the subbundle $E_{i}$ is contained in some holomorphic subbundle $\mathcal{B}_{p}$ of $E$, orthogonal to $E_{j}$. It follows that $A_{j i}^{\prime \prime}=0$, which implies also that $A_{i j}^{\prime}=0$. The case $i, j \notin \sigma$ is treated in a similar way. 
Suppose next that $i<j$ and $i \notin \sigma, j \in \sigma$. Then $E_{j}=B_{p}$ for some $B_{p} \subset$ $\mathcal{B}_{p}$. Since $E$ is harmonic, it follows that differential $d z \otimes A_{E}^{\prime}$ is holomorphic (cf. equations (8), (9) in Sec. 2.1). Here, $A_{E}^{\prime}$ is considered as a section of the holomorphic bundle $\operatorname{Hom}\left(E, E^{\perp}\right)$. Since the image $A_{E}^{\prime}\left(\mathcal{B}_{p}\right)$ is spanned by meromorphic sections of $E^{\perp}$ with divisors of degree, greater than $\delta_{j}+1$, we have

$$
A_{E}^{\prime}\left(E_{j}\right) \subset \bigoplus_{q \notin \sigma, q>j} E_{q}
$$

Hence, $A_{i j}^{\prime}=0$ for $i<j$, implying also that $A_{j i}^{\prime \prime}=0$. The case $i \in \sigma, j \notin \sigma$ is treated in a similar way, using the fact that the subbundle $E^{\perp}$ is harmonic if $E$ has this property.

3.7. Reducing the length of harmonic bundles. Consider again the HarderNarasimhan filtration (16) from Sec. 3.5.

$$
0=\mathcal{B}_{0} \subset \mathcal{B}_{1} \subset \ldots \subset \mathcal{B}_{s}=E
$$

with the quotients

$$
\mathcal{B}_{i} / \mathcal{B}_{i-1} \cong \underbrace{L^{\beta_{i}} \oplus \ldots \oplus L^{\beta_{i}}}_{b_{i} \text { times }} \cong b_{i} L^{\beta_{i}}
$$

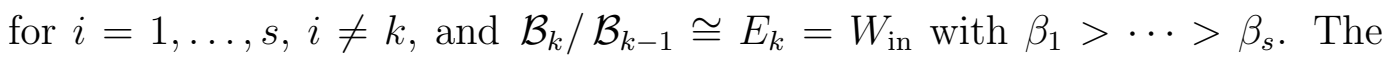
number $\beta_{1}-\beta_{s}$ is called the length of the bundle $E$.

Burstall and Salamon in [5] have proposed a procedure of reducing the length of the original bundle $E$, which is similar to Uhlenbeck's "subtracting-auniton" construction or Valli's construction, reducing the energy of a harmonic bundle. The Burstall-Salamon's procedure is immediately extended to the case of infinite-dimensional harmonic bundles. Since the argument essentially repeats the corresponding argument from [5] we formulate only the main result.

Theorem 5. For a given harmonic bundle $E \subset H \times P^{1}$ there exists a finite sequence $E^{0}, E^{1}, \ldots, E^{N}=E$ of harmonic bundles such that

(i) $0=$ length $\left(E^{0}\right)<$ length $\left(E^{1}\right)<\ldots<\operatorname{length}\left(E^{N}\right)=\beta_{1}-\beta_{s}$,

(ii) $E^{i-1}$ is obtained from $E^{i}$ or $\left(E^{i}\right)^{\perp}$ by removing a holomorphic or antiholomorphic subbundle.

According to this theorem, everything is reduced to the case of harmonic bundles of length zero which are described by the following

Proposition 1. Any harmonic bundle of length zero has the form

$$
E=F \ominus F_{1}
$$

where $F_{1}$ and $F$ are holomorphic subbundles of $\mathbb{P}^{1} \times H$, satisfying the condition $\frac{\partial}{\partial z} \Gamma\left(F_{1}\right) \subset \Gamma(F)$. 


\section{REFERENCES}

[1] M.F.Atiyah, Geometry of Yang-Mills Fields, Lezioni Fermiani.- Pisa: Scuola Normale Superiore, 1979.

[2] M.F.Atiayh, Instantons in Two and Four Dimensions, Comm. Math. Phys. 93(1984), 437-451.

[3] M.F.Atiyah, N.J.Hitchin, I.M.Singer, Self-duality in four-dimensional Riemannian geometry, Proc. Roy. Soc. London 362(1978), 425-461.

[4] A.Borel A, F.Hirzebruch, Characteristic classes and homogeneous spaces I, Amer. J. Math. 80(1958), 458-538.

[5] F.Burstall, S.Salamon, Tournaments, flags and harmonic maps, Math. Ann. 277(1987), 249-265.

[6] F.Burstall, J.Wood, The construction of harmonic maps into complex Grassmannians, J. Diff. Geom. 23(1986), 255-297.

[7] S.K.Donaldson, Instantons and geometric invariant theory, Commun. Math. Phys. 93(1984), 453-460.

[8] J.Eells, S.Salamon, Twistorial constructions of harmonic maps of surfaces into fourmanifolds, Ann. Scuola Norm. Super. Pisa 12(1985), 589-640.

[9] J.Eells, J.Sampson, Harmonic maps of Riemannian manifolds, Amer. J. Math. 86(1964), 109-160.

[10] G.Harder, M.S.Narasimhan, On the cohomology groups of moduli spaces of vector bundles over curves, Math. Ann. 212 (1975), 215-248.

[11] J.Koszul, B.Malgrange, Sur certaines structures fibrées complexes, Arch. Math. 9(1958), 102-109.

[12] A.G.Sergeev, Kähler Geometry of Loop Spaces, Moscow Centre for Continuous Math. Education, Moscow, 2001.

[13] A.G.Sergeev, Factorization of Hölder-continuous operator-functions, Uspekhi matem. nauk (Russian Math. Surveys) 27, No.6 (1972), 253 (in Russian)

[14] A.G.Sergeev, Factorization of Hölder-continuous operator-functions, Vestnik MGU (Moscow Univ. Vestnik). Ser. I: Mathematics and Mechanics 28, No.3 (1973), 58-65(in Russian)

[15] A.G.Sergeev, Harmonic spheres conjecture, Theor. Mathem. Phys. 164 (2012), 11401150 .

[16] J.Wood, The explicit construction and parametrization of all harmonic maps from the two-sphere to a complex Grassmannian, J. reine angew. math. 386(1988), 1-31.

Moscow State University, Steklov Mathematical Institute 